\title{
Assessment of antibodies against surface and outer membrane proteins of Anaplasma phagocytophilum in Lyme borreliosis and tick-borne encephalitis paediatric patients
}

\author{
L. KRBKOVÁ ${ }^{1 *}$, L. HOMOLA ${ }^{1}$, A. HLAVÁČOVÁ ${ }^{1}$, P. MIKOLÁŠEK ${ }^{1}$, \\ J. BEDNÁŘOVÁ ${ }^{2}$ AND Z. ČERMÁKOVÁ ${ }^{3}$ \\ ${ }^{1}$ Department of Children's Infectious Diseases, Faculty of Medicine and University Hospital, Masaryk \\ University, Brno, Czech Republic \\ ${ }^{2}$ Department of Clinical Microbiology, University Hospital, Brno, Czech Republic \\ ${ }^{3}$ Department of Clinical Biochemistry, University Hospital, Brno, Department of Laboratory Methods, Faculty of \\ Medicine, Masaryk University, Brno, Czech Republic
}

Received 10 September 2015; Final revision 6 April 2016; Accepted 27 April 2016;

first published online 16 May 2016

\section{SUMMARY}

To examine evidence of positive antibodies against immunogenic proteins of Anaplasma phagocytophilum in patients with other tick-borne infections and to diagnose possible coinfections, 412 serum specimens were tested by immunoblotting using three specific Anaplasma antigens: surface proteins p44 and Asp62 and outer membrane protein A (OmpA). In total, 284 serum samples from children with Lyme borreliosis and 12 serum samples from children with tick-borne encephalitis were tested. Sera from patients with viral aseptic meningitis $(n=47)$ and from blood donors $(n=69)$ were used as controls. Among all serum specimens from patients with tick-borne infections submitted for this study, six samples $(2 \cdot 0 \%)$ showed positive IgM reactions and seven samples $(2 \cdot 4 \%)$ were IgG positive for A. phagocytophilum by immunoblot. Borderline reactivity was found in 30 samples $(10 \cdot 14 \%)$ for IgM and 36 samples $(12 \cdot 2 \%)$ for IgG. The difference between patients and blood donors was statistically significant for $\operatorname{IgM}(P=0.006)$ and for $\operatorname{IgG}(P=0 \cdot 0007)$ antibodies. A statistically significant result was obtained for $\operatorname{IgG}(P=0 \cdot 02)$ but not for IgM between patients and children with aseptic meningitis. Immunoblot using three specific antigens provides novel information about the positivity of antibodies to A. phagocytophilum in children with other tick-borne infections. Taking into account clinical and laboratory findings of children despite antibody positivity, no case of human granulocytic anaplasmosis was demonstrated.

Key words: Anaplasma phagocytophilum, human granulocytic anaplasmosis, Lyme borreliosis major surface proteins, outer membrane protein A, tick-borne encephalitis.

\section{INTRODUCTION}

An increasing incidence of tick-borne diseases constitutes a serious medical problem. New pathogens that

\footnotetext{
* Author for correspondence: Dr L. Krbková, Department of Children's Infectious Diseases, Faculty of Medicine and University Hospital, Masaryk University, Jihlavská 100, 62500 Brno, Czech Republic.

(Email: lkrbkova@fnbrno.cz)
}

previously had cycled exclusively within natural zoonotic hosts have been identified as human pathogens. Anaplasma phagocytophilum, as a causative agent of human granulocytic anaplasmosis (HGA), was first described in the United States in 1994 [1]. Since 2009, the Centers for Disease Control and Prevention have declared HGA to be a notifiable disease [2]. New cases have been reported from several countries in Europe, including Slovenia, Spain, Austria, Germany, 
The Netherlands, Sweden, Denmark, Norway, Poland, Croatia and Latvia [3]. The first confirmed case of HGA in Europe was described in Slovenia [4], and the first paediatric case, in an 11-year-old girl, also in Slovenia [5]. Most cases of HGA have been contracted in geographical regions that are endemic for Lyme borreliosis (LB) and tick-borne encephalitis (TBE). Ixodes ricinus, I. scapularis, and I. persulcatus are the arthropod hosts for the causative agents of LB and TBE as well as for HGA.

A. phagocytophilum is an obligate, intracellular Gram-negative bacterium that infects granulocytes and causes a non-specific febrile illness. The usual clinical signs and symptoms include fever, chills, headache, and generalized myalgia [6]. Anaplasmosis is associated with suggestive changes in routine laboratory test parameters. Most patients develop transient reduction in total leukocyte and platelet concentrations. Characteristic intracytoplasmatic morulae are observed in peripheral blood granulocytes. Inflammatory markers, such as C-reactive protein and the erythrocyte sedimentation rate, rise during the fever period. Serum hepatic transaminases usually increase [3, 7]. Treatment with doxycycline for 10-14 days is effective, and administration of macrolides can bring about recovery in children aged $<8$ years [6].

The clinical diagnosis may be confirmed by specific laboratory tests, which include polymerase chain reaction (PCR) or cultivation. The examination of blood smears for intracytoplasmic morulae is not a sensitive approach for laboratory diagnosis, even in PCR-positive or culture-positive patients. Serological tests are not standardized. Indirect immunofluorescence assay (IFA) is performed to access fourfold increases in titres or seroconversion between paired serum specimens. The IFA-positive results are confirmed by Western blot to detect A. phagocytophilum-specific antibodies $[8,9]$.

Surface proteins of A. phagocytophilum are immunodominant antigens, and surface and outer membrane purified proteins are used as antigens in immunoblot. Protein 44 , a $\sim 44 \mathrm{kDa}$ antigen, is a major surface protein 2 (Msp2). Of the major proteins, two surface proteins Asp62 and Asp55 $(62 \mathrm{kDa}$ and $55 \mathrm{kDa}$ Anaplasma proteins) have been shown to be immunoreactive in HGA patients [10]. Outer membrane protein A (OmpA), is a peptidoglycan-associated lipoprotein [11]. OmpA plays an important role in the pathogenesis of HGA [11].

The aim of the study was to find positive antibodies against three specific Anaplasma antigens in paediatric patients with other tick-borne diseases using Western blot and in the case of positive results to search for possible co-infections.

\section{PATIENTS AND METHODS}

Altogether, 412 serum samples (284 serum samples from children with LB, 12 serum samples from children with TBE and 116 controls) were tested by immunoblot using three specific antigens derived from A. phagocytophilum.

Serum samples were selected retrospectively from 296 children with tick-borne infections (2-18 years) and 47 children with aseptic meningitis (AM) as controls (2-18 years) who had been admitted to the Department of Children's Infectious Diseases at the University Hospital, Brno, Czech Republic from 2002 to 2014. Archived serum samples were stored at $-20^{\circ} \mathrm{C}$ until used in this study. Only serum samples obtained prior to antibiotic treatment were used. At the time of sampling, no IFA testing was required with the exception of three samples, where there was clinical suspicion of HGA. Unfortunately, it was not possible to perform IFA tests and Anaplasma PCR for all samples due to small amount of material. Whole blood is required for PCR detection.

All children with Lyme neuroborreliosis (LNB) ( $n$ $=138$ ) fulfilled three criteria for the diagnosis as recommended in several guidelines: clinical signs and symptoms consistent with neuroborreliosis, lymphocytic pleocytosis (mononuclear cell count $>5 / \mu \mathrm{l}$ ) in cerebrospinal fluid (CSF), and intrathecally synthesized IgM/IgG antibodies (antibody index $>1 \cdot 4$ ) [12, 13]. Children diagnosed with TBE $(n=12)$ had demonstrable IgM tick-borne encephalitis virus (TBEV) antibodies in serum and CSF.

A Lyme arthritis (LA) group $(n=146)$ included children with clinically well-defined arthritis (a minimum of a single or repeated swelling of large joints) and high levels of specific Borrelia burgdorferi IgG antibodies. Patients with solely arthralgia and positive Lyme serology were excluded.

Sera from patients with AM $(n=47)$ and from adult blood donors $(n=69)$ were used as controls. For viral aetiology, herpetic viruses including HSV1 and HSV2, varicella zoster virus, Epstein-Barr virus, cytomegalovirus (LightCycler Qual kit, Roche Diagnostics GmbH, Germany), HHV6 (LightMix kit HHV-6 EC, TIB Molbiol GmbH, Germany), enteroviruses and adenovirus (both R-gene, Argene, France) were tested in serum and CSF by PCR. LNB and TBE were 
Table 1. Basic demographic data, duration of symptoms before sampling, and fever in patients and controls

\begin{tabular}{lllll}
\hline \hline Diagnosis & $\begin{array}{l}\text { Mean age } \\
\text { (years) }\end{array}$ & $\begin{array}{l}\text { Gender } \\
\text { (male/female) }\end{array}$ & $\begin{array}{l}\text { Median symptoms duration } \\
\text { at presentation in days (range) }\end{array}$ & $\begin{array}{l}\text { Patients } \\
\text { with fever }(\%)\end{array}$ \\
\hline LNB $(n=138)$ & $10 \cdot 9 \pm 4 \cdot 5$ & $60 / 78$ & $7(1-120)$ & $20 \cdot 2$ \\
LA $(n=146)$ & $12 \cdot 9 \pm 3 \cdot 9$ & $87 / 59$ & $61(15-1459)$ & $1 \cdot 4$ \\
TBE $(n=12)$ & $11 \cdot 5 \pm 4 \cdot 1$ & $7 / 5$ & $12 \cdot 5(3-19)$ & $100 \cdot 0$ \\
AM $(n=47)$ & $10 \cdot 1 \pm 4 \cdot 8$ & $29 / 18$ & $3(1-21)$ & $87 \cdot 2$ \\
Blood donors $(n=69)$ & Adults & Unknown & n.a. & n.a. \\
\hline
\end{tabular}

LNB, Lyme neuroborreliosis; LA, Lyme arthritis; TBE, tick-borne encephalitis; AM, aseptic meningitis; n.a., not applicable.

excluded in the one group of 47 children with AM. Enteroviral meningitis was diagnosed in 20 children, once HSV1, HHV6 and adenovirus were found in CSF. No causative agent was detected in 24 children.

Samples from adult blood donors were obtained through the hospital service. Testing for tick-borne infections was not performed. Blood donors were asked about diagnosed tick-borne infections or clinical symptoms after a tick bite in their history in an obligatory questionnaire.

Demographic characteristics, duration of symptoms at the time of sampling and fever are presented in Table 1. Any symptoms and signs, i.e. headache, malaise, vomiting, fever, facial palsy, joint swelling, etc. which were reported by the parents were included in the evaluation.

Medical history of all children with positive or borderline reactivity to Anaplasma antigens was evaluated for possible HGA infection by checking for tick-bite history, clinical signs and symptoms compatible with HGA, and typical laboratory findings such as leukopenia (normal value $4 \cdot 5-13.5 \times 10^{9} / 1$ ), thrombocytopenia (normal value $150-450 \times 10^{9} / 1$ ) and elevated liver transaminases [alanine transaminase (ALT) normal value $0 \cdot 2-0 \cdot 6 \mu \mathrm{kat} / \mathrm{l}$, aspartate transaminase (AST) normal value $0 \cdot 2-0.63 \mu \mathrm{kat} / 1]$.

The tested immunoblot (BLOT-LINE Anaplasma IgG/IgM; TestLine Clinical Diagnostics, Czech Republic) is based on highly specific recombinant Anaplasma antigens known to be proteins with high immunoreactivity in HGA patients (p44, Asp62, OmpA) and applied on a nitrocellulose membrane. In the first reaction step, as the individual strips are incubated $(30 \mathrm{~min})$ with the tested samples (diluted 1:51), the specific antibodies (if present in the sample) bind to the corresponding antigenic bands on the strip. After washing, the strips are further incubated with a conjugate (alkaline phosphatase). Visualization is achieved by incubation with a substrate solution (5-bromo-4-chloro-3-indolyl phosphate with nitroblue tetrazolium chloride). After colour development, the test strips are dried and evaluated. For test validity verification, the strips are provided with a conjugate control band and with a control band indicating kit functionality and sensitivity. Testing was performed according to the manufacturer's instructions.

The evaluation was done using software (Immunoblot Software v. 1.3.7; TestLine Clinical Diagnostics). Test evaluation is based on the combined presence of the specific antigenic bands and their intensity (percentage value of band intensity). The intensity is measured in the range of $0-100 \%$. The cut-off range is precisely defined to be $5 \cdot 5-13.0 \%$. Values falling within this range are classified as borderline. Negative results are those with a value $<5.5 \%$ while positive results are those with a value $>13 \cdot 0 \%$. All three antigens mentioned above (p44, Asp62, OmpA) are applied on the same strip and are detected in the same reaction steps. Results are considered to be positive when at least one of specific antigens is positive (the intensity of antigenic line $>13.0 \%$ ) and simultaneously at least one other of the specific antigens is borderline (the intensity of the antigenic line is in the range $5 \cdot 5-13 \cdot 0 \%$ ).

The panels of positive and negative sera with defined clinical picture or panels with defined presence of antibodies where the positivity and negativity was known from other independent sources were tested. Cut-off is a threshold between the negative and positive samples. The use of the cut-off range can solve the problem of overlapping results. The specified cutoff range was $5 \cdot 5-13.0 \%$. The minimum of the cut-off range was determined by evaluation of the diagnostic sensitivity (D-SN) of the test whereas the maximum of the cut-off range was determined from analysis of the diagnostic specificity (D-SP) of the test.

\section{Statistical analysis}

The data were analysed using $\mathrm{R}$ software $\mathrm{v}$. 3.1.3 (https://cran.r-project.org/bin/windows/base/old/3.1.3/). 
Table 2. IgM and IgG reactivity of 12 patients' serum specimens against three Anaplasma phagocytophilum antigens used in immunoblot assay

\begin{tabular}{|c|c|c|c|c|c|c|c|c|c|}
\hline \multirow[b]{2}{*}{ Patient no. } & \multirow[b]{2}{*}{ Diagnosis } & \multicolumn{4}{|l|}{$\operatorname{IgM}$} & \multicolumn{4}{|l|}{$\mathrm{IgG}$} \\
\hline & & p44 \% & Asp62 \% & OmpA \% & IgM evaluation & p44 \% & Asp62\% & OmpA $\%$ & IgG evaluation \\
\hline 132 & LNB & $0 \cdot 8$ & $2 \cdot 4$ & $2 \cdot 0$ & Neg. & $22 \cdot 9$ & $10 \cdot 0$ & $10 \cdot 8$ & Pos. \\
\hline 33 & LNB & $2 \cdot 6$ & $1 \cdot 6$ & $3 \cdot 0$ & Neg. & $14 \cdot 6$ & $8 \cdot 4$ & $4 \cdot 9$ & Pos. \\
\hline 17 & LNB & $13 \cdot 7$ & $9 \cdot 4$ & $12 \cdot 9$ & Pos. & $0 \cdot 8$ & $3 \cdot 5$ & $0 \cdot 4$ & Neg. \\
\hline 94 & LNB & $7 \cdot 8$ & $5 \cdot 5$ & $15 \cdot 3$ & Pos. & $2 \cdot 0$ & $3 \cdot 9$ & $0 \cdot 0$ & Neg. \\
\hline 185 & LNB & $13 \cdot 7$ & $4 \cdot 3$ & $6 \cdot 3$ & Pos. & $0 \cdot 0$ & $0 \cdot 4$ & $0 \cdot 4$ & Neg. \\
\hline 104 & LNB & $2 \cdot 6$ & $4 \cdot 2$ & $4 \cdot 9$ & Neg. & $4 \cdot 0$ & $11 \cdot 1$ & $13 \cdot 5$ & Pos. \\
\hline 58 & LNB & $2 \cdot 3$ & $0 \cdot 6$ & $1 \cdot 9$ & Neg. & $60 \cdot 6$ & $5 \cdot 6$ & $4 \cdot 0$ & Pos. \\
\hline 5 & LNB & $1 \cdot 4$ & $1 \cdot 4$ & $0 \cdot 8$ & Neg. & $68 \cdot 2$ & $7 \cdot 4$ & $5 \cdot 8$ & Pos. \\
\hline 15 & LA & $25 \cdot 7$ & $9 \cdot 1$ & $4 \cdot 3$ & Pos. & $8 \cdot 2$ & $5 \cdot 6$ & $2 \cdot 6$ & Bor. \\
\hline 62 & LA & $23 \cdot 5$ & $8 \cdot 7$ & $4 \cdot 7$ & Pos. & $9 \cdot 3$ & $8 \cdot 7$ & $5 \cdot 8$ & Bor. \\
\hline 82 & LA & $42 \cdot 5$ & $3 \cdot 8$ & $5 \cdot 6$ & Pos. & $22 \cdot 1$ & $9 \cdot 6$ & $6 \cdot 2$ & Pos. \\
\hline 92 & LA & $5 \cdot 1$ & $0 \cdot 8$ & $4 \cdot 7$ & Neg. & $9 \cdot 0$ & $6 \cdot 3$ & $13 \cdot 3$ & Pos. \\
\hline
\end{tabular}

LNB, Lyme neuroborreliosis, LA, Lyme arthritis, Neg., negative $(<5 \cdot 5 \%)$, Pos., positive ( $>13 \cdot 0 \%)$, Bor., borderline $(5 \cdot 5-13 \cdot 0 \%)$.

For categorical data, comparison was made using Fisher's exact test or $\chi^{2}$ test. Statistical significance was considered as $P<0 \cdot 05$.

\section{RESULTS}

Of all serum specimens from patients with tick-borne infections submitted for this study, six samples $(2 \cdot 0 \%)$ showed positive IgM reactions and seven samples $(2 \cdot 4 \%)$ were positive in $\mathrm{IgG}$ for $A$. phagocytophilum by immunoblot in 12 children (Table 2). One child with LA characterized solely by a swollen knee joint was febrile with headache, malaise and myalgia, symptoms which can be attributed more to HGA than to LA. The 11-year-old boy developed joint pain and a moderate elevation of temperature 17 days prior to admission. The leukocyte and thrombocyte cell count $\left(8.66 \times 10^{9} / 1,298 \times 10^{9} / 1\right)$ revealed normal values. The liver enzymes were not increased (ALT $0.45 \mu \mathrm{kat} / \mathrm{l}$, AST $0.58 \mu \mathrm{kat} / \mathrm{l}$ ). The C-reactive protein was $<1 \mathrm{mg} / \mathrm{l}$. No lumbar puncture was performed. That patient's serum specimen (no. 82, Table 2) reacted against two antigens in $\operatorname{IgM}(\mathrm{p} 44$ and OmpA) and against three antigens in IgG (p44, Asp62, OmpA).

Eight serum specimens were found positive by immunoblot in children with LNB, and four serum specimens revealed positivity for HGA in children with LA (reactivity against Anaplasma antigens are shown in Table 2). Total leukocyte and platelet concentrations and serum hepatic transaminases were within the reference range in all 12 children (for reference range see Patients and methods section). Despite seropositive results for HGA, none of the children could be diagnosed as having co-infection because they did not fulfil clinical and laboratory criteria for correct diagnosis of HGA. All children were treated for borrelial infection with ceftriaxone 14 days for LNB and 28 days for LA.

Borderline reactivity was found in 30 samples $(10 \cdot 1 \%)$ for $\operatorname{IgM}$ and 36 samples $(12 \cdot 2 \%)$ for $\operatorname{IgG}$. Table 3 shows the statistical significance of patients and both control groups for pooled borderline and positive results. The pooling was done to avoid small number errors in Fisher's exact test. A statistically significant result was obtained for $\operatorname{IgG}(P=0.02)$ between patients and children with AM. The differences reached statistical significance for $\operatorname{IgM}(P=$ $0 \cdot 006)$ as well as for $\operatorname{IgG}(P=0.0007)$ antibodies comparing patients with blood donors.

There was no difference between patients and the AM group of children based on all categories either in $\operatorname{IgM}$ or $\operatorname{IgG}$, only between patients and blood donors (IgM: $P=0.03$; IgG: $P=0 \cdot 003$ ).

IgM antibodies were positive against $\mathrm{p} 44$ antigen in $40(13.5 \%)$ serum specimens of children with tickborne infections. Reaction with two specific antigens was defined as positive in $17(5.7 \%)$ serum specimens and with all three Anaplasma antigens in seven $(2 \cdot 4 \%)$ serum specimens (Table 4$)$.

When purified Anaplasma antigen p44 (Msp2) was used, $56(18.9 \%)$ serum specimens showed positive or borderline reaction for IgG in the LB group of 
Table 3. IgM and IgG results of all patients' serum specimens $(n=412)$ vs. group of children with aseptic meningitis ( AM) and blood donors. Specificity is $94.8 \%$ for IgM, $100 \%$ for $\operatorname{Ig} G$

\begin{tabular}{lllll}
\hline \hline Antibody class & Evaluation & Patients $(n=296)$ & AM $(n=47)$ & Blood donors $(n=69)$ \\
\hline IgM & Negative & $260(87 \cdot 9 \%)$ & $42(89 \cdot 4 \%)$ & $68(98 \cdot 6 \%)$ \\
& Borderline & $30(10 \cdot 1 \%)$ & $4(8 \cdot 5 \%)$ & $1(1 \cdot 4 \%)$ \\
& Positive & $6(2 \cdot 0 \%)$ & $1(2 \cdot 1 \%)$ & $0(0 \cdot 0 \%)$ \\
\hline
\end{tabular}

$\chi^{2}$ test: $P=0.90$ (patients $v s$. AM group), $P=0.03$ (patients $v s$. blood donors)

Fisher's test: $P=\sim 1$ (patients $v s$. AM group), $P=0.006$ (patients $v s$. blood donors)

\begin{tabular}{lllll}
\hline Antibody class & Evaluation & Patients $(n=296)$ & AM $(n=47)$ & Blood donors $(n=69)$ \\
\hline $\mathrm{IgG}$ & Negative & $253(85 \cdot 4 \%)$ & $47(100 \cdot 0 \%)$ & $69(100 \cdot 0 \%)$ \\
& Borderline & $36(12 \cdot 2 \%)$ & $0(0 \cdot 0 \%)$ & $0(0 \cdot 0 \%)$ \\
& Positive & $7(2 \cdot 4 \%)$ & $0(0 \cdot 0 \%)$ & $0(0 \cdot 0 \%)$ \\
\hline
\end{tabular}

$\chi^{2}: P=0.06$ (patients vs. AM group), $P=0.003$ (patients $v s$. blood donors)

Fisher's test: $P=0.02$ (patients $v s$. AM group), $P=0.0007$ (patients $v s$. blood donors)

Negative, Samples with intensity $<5 \cdot 5 \%$; Positive, samples with intensity $>13 \cdot 0 \%$; Borderline, samples with intensity in the range $5 \cdot 5-13 \cdot 0 \%$.

$\chi^{2}$ test based on all categories.

Fisher's exact test based on pooled borderline plus positive values due to small numbers.

Table 4. IgM results: reactivity against one of the group of Anaplasma phagocytophilum antigens ( $p 44$, Asp62, OmpA); reactivity against two of the mentioned antigens simultaneously, and reactivity against all three

A. phagocytophilum antigens in patients with tick-borne infections, patients with aseptic meningitis and blood donors

\begin{tabular}{|c|c|c|c|c|c|}
\hline $\operatorname{IgM}$ & Samples & Negative & 1 antigen & 2 antigens & 3 antigens \\
\hline LNB & 138 & $102(73 \cdot 9 \%)$ & $19(13 \cdot 8 \%)$ & $11(8 \cdot 0 \%)$ & $6(4 \cdot 3 \%)$ \\
\hline LA & 146 & $122(83 \cdot 6 \%)$ & $17(11 \cdot 6 \%)$ & $6(4 \cdot 1 \%)$ & $1(0 \cdot 7 \%)$ \\
\hline TBE & 12 & $8(66 \cdot 7 \%)$ & $4(33 \cdot 3 \%)$ & $0(0 \cdot 0 \%)$ & $0(0 \cdot 0 \%)$ \\
\hline $\mathrm{AM}$ & 47 & $36(76 \cdot 6 \%)$ & $8(17 \cdot 0 \%)$ & $3(6 \cdot 4 \%)$ & $0(0 \cdot 0 \%)$ \\
\hline Blood donors & 69 & $67(97 \cdot 1 \%)$ & $2(2 \cdot 9 \%)$ & $0(0 \cdot 0 \%)$ & $0(0 \cdot 0 \%)$ \\
\hline
\end{tabular}

LNB, Lyme neuroborreliosis; LA, Lyme arthritis; TBE, tick-borne encephalitis; AM, aseptic meningitis; Negative, samples with intensity $<5 \cdot 5 \%$.

children. From these, $19(6 \cdot 4 \%)$ and $10(3 \cdot 4 \%)$ serum specimens reacted against two and three Anaplasmaspecific antigens, respectively (Table 5).

Borderline and positive results for IgM and $\mathrm{IgG}$ would reach $32 \cdot 4 \%(96 / 296)$ if only antibodies against p44 were evaluated.

Indirect IFA tests were performed on the sera of three children during standard examination after a tick bite at the time of admission. HGA was suspected clinically (headache, fever, fatigue) and from laboratory results due to leukopenia (leukocyte cell count from $2 \cdot 7$ to $4 \cdot 4 \times 10^{9} / 1$ ), thrombocytopenia (thrombocyte cell count from 54 to $\left.133 \times 10^{9} / 1\right)$, and elevated hepatic enzymes (ALT 0.8-5.19 $\mu \mathrm{kat} / \mathrm{l}$, AST 1.2-7.3 $\mu \mathrm{kat} / \mathrm{l}$ ). IFA revealed no increased levels of Anaplasma-specific antibodies. All three samples were tested negative by immunoblot with three specific Anaplasma antigens. The children were confirmed as having TBE. Convalescent serum samples tested by IFA were negative, but were not available for testing by immunoblot.

Western blotting results obtained from the AM group of children in IgM, were borderline in four samples $(8.5 \%)$ and positive in one sample $(2 \cdot 1 \%)$ (Table 3). One sample $(1 \cdot 4 \%)$ showed borderline 
Table 5. IgG results: reactivity against one of the group of Anaplasma phagocytophilum antigens (p44, Asp62, OmpA); reactivity against two of the mentioned antigens simultaneously, and reactivity against all three

A. phagocytophilum antigens in patients with tick-borne infections, patients with aseptic meningitis and blood donors

\begin{tabular}{lllcrl}
\hline \hline IgG & Samples & Negative & 1 antigen & 2 antigens & 3 antigens \\
\hline LNB & 138 & $101(73 \cdot 2 \%)$ & $27(19 \cdot 6 \%)$ & $8(5 \cdot 8 \%)$ & $2(1 \cdot 4 \%)$ \\
LA & 146 & $98(67 \cdot 1 \%)$ & $29(19 \cdot 9 \%)$ & $11(7 \cdot 5 \%)$ & $8(5 \cdot 5 \%)$ \\
TBE & 12 & $12(100 \cdot 0 \%)$ & $0(0 \cdot 0 \%)$ & $0(0 \cdot 0 \%)$ & $0(0 \cdot 0 \%)$ \\
AM & 47 & $40(85 \cdot 1 \%)$ & $7(14 \cdot 9 \%)$ & $0(0 \cdot 0 \%)$ & $0(0 \cdot 0 \%)$ \\
Blood donors & 69 & $62(89 \cdot 9 \%)$ & $7(10 \cdot 1 \%)$ & $0(0 \cdot 0 \%)$ & $0(0 \cdot 0 \%)$ \\
\hline \hline
\end{tabular}

LNB, Lyme neuroborreliosis; LA, Lyme arthritis; TBE, tick-borne encephalitis; AM, aseptic meningitis; Negative, samples with intensity $<5 \cdot 5 \%$.

reactivity in IgM antibodies in the blood donor group. No sample was positive in IgG antibodies either in the AM group of children or in blood donors.

Taking all sera from children with AM and adult blood donors together, the specificity of the used immunoblot with three specific Anaplasma antigens was $94 \cdot 8 \%$ for $\operatorname{IgM}$ antibodies and $100 \%$ for IgG antibodies.

\section{DISCUSSION}

Seroprevalence of Anaplasma antibodies in human sera has been investigated in several European studies and positivity has ranged from low percentages of $1.4 \%$ and $2 \cdot 8 \%$ to extremely high at $28 \%$ [14-18]. Antibodies to HGA were found, using IFA ( $\geqslant 1: 160)$, to occur in $13.5 \%$ [19] or using immunoblot assay in $10 \cdot 8 \%$ of adult patients with suspected LB [20]. In the tick vector collected from humans, the prevalence of A. phagocytophilum is $1.5 \%$ in Prague parks, Czech Republic. Ticks collected by flagging were infected by Anaplasma in $11.8 \%$ of samples [21].

No study to date has demonstrated antibodies to $A$. phagocytophilum in children with proven tick-borne infections in the Czech Republic. To examine this more closely, children with diagnoses of LNB, LA and TBE were selected for serological evaluation of antibodies. LNB in children is usually an early disseminated disease with $\operatorname{IgM} / \operatorname{IgG}$ antibody response to $B$. burgdorferi, while LA is a late-disseminated infection accompanied by a strong IgG response [13, 2224]. TBE in children is an acute AM/encephalitis with a maximum incubation period of 3 weeks after a tick bite, similar to HGA. Three children diagnosed as TBE were suspected of HGA in the febrile phase of the illness. Their laboratory findings showed leukopenia, thrombocytopenia and elevated liver enzymes. Similar laboratory abnormalities were found in TBE patients [25]. Immunoblot test with three specific antigens of $A$. phagocytophilum detected no antibodies. IFA was performed in routine testing with negative results. Both infections should be taken into consideration in clinical practice.

The proportion of $A$. phagocytophilum serum antibodies detected by immunoblot using three specific antigens was $2 \cdot 0 \%$ for IgM and $2 \cdot 4 \%$ for IgG. The positivity to specific antigens of $A$. phagocytophilum was low in children with proven tick-borne infections. Only for one child was there a clinical correlation to HGA, and that child had been admitted with fever, headache and myalgia. Hepatic enzymes, leukocytes and platelet concentrations were within the reference limit. PCR was not performed. The child was diagnosed with LA and effectively treated by ceftriaxone.

Differences in the detection of positively reacting antibodies against three specific Anaplasma antigens between the TBE group of children with tick-borne infections and blood donors were found to be statistically significant (IgM: $P=0 \cdot 006 ; \operatorname{IgG}: P=0 \cdot 0007$ ) based on pooled borderline and positive values. A significantly higher number of $A$. phagocytophilumreactive sera in the patient group compared to the AM group of children in IgG class may be due to anamnestic antibodies. This finding probably reflects previous asymptomatic $A$. phagocytophilum infection. It is generally known that IgM antibodies to A. phagocytophilum are less specific than IgG antibodies and result in more false positives. Higher frequency of IgM antibodies were observed in LB patients vs. blood donors in Southern Germany [26].

In data from Switzerland, the highest prevalence of A. phagocytophilum antibodies occurred in people who were seropositive for B. burgdorferi $(13 \%)$ and TBEV (20\%) [27]. In Slovenia, where all three infections are endemic, the healthy population of children and young adults was assessed for the presence of antibodies to B. burgdorferi, A. phagocytophilum and 
TBEV. An identical percentage of antibodies (15.4\%) to A. phagocytophilum and B. burgdorferi, respectively, was detected [15].

To compare our results with similar European studies is problematical even if only immunoblot is used. Enrolment criteria for patients are not identical and several antigens are used in commercially available immunoblots. Serological evidence of HGA infection investigated by IFA in LB patients $(n=43)$ has been confirmed in one individual $(2 \cdot 3 \%)$ with previous neuroborreliosis in a study from northern Spain [14]. In a study by Lebech et al., antibodies to Ehrlichia equi (now renamed $A$. phagocytophilum) were assayed by IFA in the sera of 132 patients with LNB. Reactivity was found in five patients $(3.8 \%)$ of whom two had clinical symptoms of fever, headache and myalgia compatible with the probable case definition of HGA [18]. Our results correlate with the last two mentioned studies of patients with proven LB, although different tests were used.

An assessment of cut-off value and assay validation is part of the development of the new assay. There is never a clear border between negative and positive results and cut-off range helps to achieve balance between D-SP and D-SN. It has to be taken into account that the cut-off range for tested immunoblot assay is exactly defined between $5.5 \%$ and $13 \%$. The values falling between the cut-off range are classified as borderline, and would require testing by a confirmatory assay or retesting.

Serum samples from patients with HGA cross-react with $E$. chaffeensis, E. ewingii and Orientia tsutsugamushi $[9,28]$. False-positive IgM response to B. burgdorferi has been recognized to occur in patients with response to A. phagocytophilum [29]. Positive reaction to A. phagocytophilum in IgM was present in three samples of children with LA and in three samples of children with LNB, but the reaction was negative if tested against Borrelia antigens in IgM. We conclude that a false-positive IgM response has not occurred between Borrelia and Anaplasma antigens.

B. burgdorferi, A. phagocytophilum and TBEV have overlapping epidemiology and transmission cycles with shared tick vectors. Infections caused by these agents may have similar clinical symptomatology in their initial phases. Searching for co-infections, Horowitz et al. [30] studied erythema migrans patients and non-specific febrile illness with potential tick exposure $(n=311)$. Positive blood culture for A. phagocytophilum was found in $2.3 \%$ and high antibody titre/ fourfold rise in titre was detected in $10 \%$ of patients with erythema migrans. The total number of clinical symptoms did not differ between patients with HGA alone and those who were co-infected. No information about disseminated LB is given in that study [30].

In order to identify possible co-infection with LB, TBE and HGA, antibodies to A. phagocytophilum were examined by immunoblot using three specific antigens. The present study investigating archived serum samples of 296 children and 116 controls was limited by an insufficient amount of sera to perform IFA and immunoblot simultaneously. However, immunoblot is generally thought to provide more detailed information than IFA $[8,9,28]$. No paired samples were available for performing seroconversion or antibody rise titre. Another disadvantage is the retrospective review of medical charts, because some less important information could not be obtained, e.g. unrelated antibiotic treatment during the last 3 months.

The emerging pathogen infection anaplasmosis caused by $A$. phagocytophilum has become a notifiable disease in the Czech Republic, as elsewhere in Europe. As shown in this study, co-infection with A. phagocytophilum in children with other tick-borne diseases has not been proven by immunoblot with three specific antigens derived from $A$. phagocytophilum. The diagnosis of HGA is currently based on PCR testing or on investigating paired serum samples by IFA. To differentiate an on-going infection from previous exposure to $A$. phagocytophilum, IgM and $\operatorname{IgG}$ antibodies tested by immunoblot with specific antigens could be used.

The results of this study have shown a small number of positive Anaplasma antibodies in children with LB and TBE. We do not recommend routine testing for HGA in children diagnosed with these two tick-borne infections even in tick-infested areas. More prospective studies comparing IFA, immunoblot and PCR detection of A. phagocytophilum are needed in children with tick-borne infections. The sensitivity and specificity of the new immunoblot with three specific antigens derived from A. phagocytophilum must be evaluated.

\section{ACKNOWLEDGEMENTS}

We thank Iva Stoklaskova, RNDr and Lenka Pokorna, RNDr, PhD, from TestLine, Clinical Diagnostics, Brno, Czech Republic for donating the immunoblot test kits with three specific antigens of Anaplasma phagocytophilum for this study and Michal Kýr, MD, PhD, for assistance with statistical analysis. 


\section{DECLARATION OF INTEREST}

None.

\section{REFERENCES}

1. Chen S, et al. Identification of a granulocytotropic Ehrlichia species as the etiologic agent of human disease. Journal of Clinical Microbiology 1994; 32: 589.

2. Adams DA, et al. Reports of nationally notifiable infectious diseases-United States, 2011. Morbidity and Mortality Weekly Report 2013; 60: 1-117.

3. Blanco JR, Oteo JA. Human granulocytic ehrlichiosis in Europe. Clinical Microbiology and Infection 2002; 8: 763-772.

4. Petrovec M, et al. Human disease in Europe caused by a granulocytic Ehrlichia species. Journal of Clinical Microbiology 1997; 35: 1556-1559.

5. Arnež M, et al. First European pediatric case of human granulocytic ehrlichiosis. Journal of Clinical Microbiology 2001; 39: 4591-4592.

6. Bakken JS, et al. Clinical and laboratory characteristics of human granulocytic ehrlichiosis. Journal of the American Medical Association 1996; 275: 199-205.

7. Lotric-Furlan S, et al. Human granulocytic ehrlichiosis in Europe: clinical and laboratory findings for four patients from Slovenia. Clinical Infectious Diseases 1998; 27: 424-428.

8. Heo E, et al. Serologic and molecular detection of Ehrlichia chaffeensis and Anaplasma phagocytophila (human granulocytic ehrlichiosis agent) in Korean patients. Journal of Clinical Microbiology 2002; 40: 3082-3085.

9. Park J, et al. Detection of antibodies to Anaplasma phagocytophilum and Ehrlichia chaffeensis antigens in sera of Korean patients by Western immunoblotting and indirect immunofluorescence assays. Clinical and Diagnostic Laboratory Immunology 2003; 10: 1059-1064.

10. Ge Y, Rikihisa Y. Identification of novel surface proteins of Anaplasma phagocytophilum by affinity purification and proteomics. Journal of Bacteriology 2007; 189: 7819-7828.

11. Ojogun $\mathbf{N}$, et al. Anaplasma phagocytophilum outer membrane protein A interacts with sialylated glycoproteins to promote infection of mammalian host cells. Infection and Immunity 2012; 80: 3748-3760.

12. Mygland A, et al. EFNS guidelines on the diagnosis and management of European Lyme neuroborreliosis. European Journal of Neurology 2010; 17: 8-16.

13. Stanek G, et al. Lyme borreliosis: clinical case definition for diagnosis and management in Europe. Clinical Microbiology and Infection 2011; 17: 79-79.

14. Oteo JA, et al. Presence of granulocytic ehrlichia in ticks and serological evidence of human infection in La Rioja, Spain. Epidemiology and Infection 2001; 127: 353-358.

15. Cizman M, et al. Seroprevalence of ehrlichiosis, Lyme borreliosis and tick-borne encephalitis infections in children and young adults in Slovenia. Wiener Klinische Wochenschrift 2000; 112: 842-845.
16. Cinco M, et al. Detection of HGE agent-like Ehrlichia in Ixodes ricinus ticks in northern Italy by PCR. Wiener Klinische Wochenschrift 1998; 110: 898-900.

17. Jenkins A, et al. Borrelia burgdorferi sensu lato and Ehrlichia sp. in Ixodes ticks from southern Norway. Journal of Clinical Microbiology 2001; 39: 3666-3671.

18. Lebech AM, et al. Immunoserologic evidence of human granulocytic ehrlichiosis in Danish patients with Lyme neuroborreliosis. Scandinavian Journal of Infectious Diseases 1998; 30: 173-176.

19. Zeman $\mathbf{P}$, et al. Antibodies to granulocytic Ehrlichiae in the population of the western and central part of the Czech Republic [in Czech, English abstract available]. Epidemiologie, Mikrobiologie, Imunologie 2002; 51: 13-18.

20. Dvořáková Heroldová M, Dvořáčková M. Seroprevalence of Anaplasma phagocytophilum in patients with suspected Lyme borreliosis [in Czech, English abstract available]. Epidemiologie, Mikrobiologie, Imunologie 2014; 63: 297-302.

21. Hulínská D, et al. Detection of Borrelia, Anaplasma, Bartonella and Rickettsia spp. in ticks Ixodes ricinus from Prague parks in 2007 and 2008 [in Czech, English abstract available]. Zprávy Centra Epidemiologie a Mikrobiologie 2009; 18: 167-171.

22. Wormser GP, et al. The clinical assessment, treatment, and prevention of Lyme disease, human granulocytic anaplasmosis and babesiosis: clinical practice guidelines by the Infectious Disease Society of America. Clinical Infectious Diseases 2006; 43: 1089-1134.

23. Steere AC, et al. Prospective study of serologic tests for Lyme disease. Clinical Infectious Diseases 2008; 47: 188-195.

24. DeBiasi RL. A concise critical analysis of serologic testing for the diagnosis of Lyme disease. Current Infectious Disease Reports 2014; 16: 450.

25. Lotrič-Furlan S, et al. Epidemiological, clinical and laboratory distinction between human granulocytic ehrlichiosis and the initial phase of tick-borne encephalitis. Wiener Klinische Wochenschrift 2002; 114: 636-640.

26. Fingerle $\mathbf{V}$, et al. Human granulocytic ehrlichiosis in Southern Germany: increased seroprevalence in highrisk group. Journal of Clinical Microbiology 1997; 35: 3244-3247.

27. Pusterla N, et al. Serological evidence of human granulocytic ehrlichiosis in Switzerland. European Journal of Clinical Microbiology and Infectious Diseases 1998; 17: 207-209.

28. Unver A, et al. Western blot analysis of sera reactive to human monocytic ehrlichiosis and human granulocytic ehrlichiosis agents. Journal of Clinical Microbiology 2001; 39: 3982-3986.

29. Wormser GP, et al. Positive Lyme disease serology in patients with clinical and laboratory evidence of human granulocytic ehrlichiosis. American Journal of Clinical Pathology 1997; 107: 142-147.

30. Horowitz HW, et al. Lyme disease and human granulocytic anaplasmosis coinfection: impact of case definition on coinfection rates and illness severity. Clinical Infectious Diseases 2013; 56: 93-99. 